\title{
Erratum: Experimental Certification of Sustained Entanglement and Nonlocality after Sequential Measurements [Phys. Rev. Applied 13, 044008 (2020)]
}

\author{
Giulio Foletto $\odot,{ }^{1}$ Luca Calderaro $\odot,{ }^{1}$ Armin Tavakoli, ${ }^{2}$ Matteo Schiavon $\odot,{ }^{1}$ Francesco Picciariello $\odot,{ }^{1}$ \\ Adán Cabello®, ${ }^{3,4}$ Paolo Villoresi ${ }^{1,5}$ and Giuseppe Vallone ${ }^{1,6}$ \\ ${ }^{1}$ Dipartimento di Ingegneria dell'Informazione, Università di Padova, IT-35131 Padova, Italy \\ ${ }^{2}$ Département de Physique Appliquée, Université de Genève, CH-1211 Genève, Switzerland \\ ${ }^{3}$ Departamento de Física Aplicada II, Universidad de Sevilla, E-41012 Sevilla, Spain \\ ${ }^{4}$ Instituto Carlos I de Física Teórica y Computacional, Universidad de Sevilla, E-41012 Sevilla, Spain \\ ${ }^{5}$ Istituto di Fotonica e Nanotecnologie, CNR, IT-35131 Padova, Italy \\ ${ }^{6}$ Dipartimento di Fisica e Astronomia, Università di Padova, IT-35131 Padova, Italy
}

(Received 22 May 2020; published 25 June 2020)

\section{DOI: 10.1103/PhysRevApplied.13.069902}

Due to a technical error in our numerical analysis computer program, in the sentence (end of Section II)

For instance, with a short sequence of two measurements, with $\mu_{1}=0.13$ and $\mu_{2}=0$, she would certify 1.026 bits

we gave the value 1.026 instead of the correct one of 0.6. We would like to change the statement to the following one to recover its intended meaning:

For instance, using the modified form of $S_{\mathrm{CHSH}}$ proposed in Ref. [18], even with a short sequence of two measurements, she could certify a number of random bits per entangled pair that is arbitrarily close to two.

The main point of the paper, i.e., the experimental demonstration that weak measurements can sustain entanglement and at the same time certify it via the violation of a Bell inequality, is not affected by this error.

We would also like to point out a minor typo in Eq. (A5): all instances of $\mu_{1}$ should be replaced by $\mu_{k}$. The correct expression is as follows:

$$
\begin{aligned}
& K_{+1 \mid 0}\left(\mu_{k}\right)=\cos \left(\mu_{k}\right)|0\rangle\left\langle 0\left|+\sin \left(\mu_{k}\right)\right| 1\right\rangle\langle 1|, \\
& K_{-1 \mid 0}\left(\mu_{k}\right)=\sin \left(\mu_{k}\right)|0\rangle\left\langle 0\left|+\cos \left(\mu_{k}\right)\right| 1\right\rangle\langle 1|, \\
& K_{+1 \mid 1}\left(\mu_{k}\right)=\cos \left(\mu_{k}\right)|+\rangle\left\langle+\left|+\sin \left(\mu_{k}\right)\right|-\right\rangle\langle-|, \\
& K_{-1 \mid 1}\left(\mu_{k}\right)=\sin \left(\mu_{k}\right)|+\rangle\left\langle+\left|+\cos \left(\mu_{k}\right)\right|-\right\rangle\langle-| .
\end{aligned}
$$

